\title{
Shining a light on the peer review process
}

\author{
Our authors now have the option to publish the review reports alongside their manuscript.
}

W e at Nature Computational
Science are committed to
providing the best possible service to both reviewers and authors by improving on the traditional peer review process. As an example of this commitment, our published manuscripts are accompanied by a reviewer recognition statement, in which reviewers can opt in to have their names well-deservedly recognized for their efforts. Furthermore, because computational science research depends on software execution, the source code associated with a manuscript is peer reviewed at our journal by at least one reviewer, as we want to ensure that our papers meet the highest standards of reproducibility and transparency in reporting. We are now incorporating one more initiative that we hope our community will be particularly excited about: transparent peer review.

Before publication, our manuscripts go through two or three rounds of review, which also include comments on the source code and to what extent the results are reproducible. We then carefully consider all of the arguments raised by reviewers to make informed decisions on whether to publish a paper. The reviews also help us to identify what authors need to work on to better report and explain their research to the community. Traditionally, these reviews are kept confidential among editors, reviewers and authors, even though they are essential to the dissemination of research.

Nature Computational Science now offers the option of transparent peer review for new original research manuscripts submitted starting from 17 February 2021. When a manuscript is accepted in principle for publication, authors will be asked whether they would like to opt in for transparent peer review. If they do, reviewers' comments, authors' rebuttal and editorial decision letters will be compiled and published alongside the paper.

Reviewers are made aware, before replying to our review invitation, that their reports may be published depending on the authors' choice. It is worth noting that the identity of the reviewers is never revealed, unless they decide to sign their reviews.

Transparent peer review is certainly not new to the Nature Portfolio family. Nature Communications implemented this process in $2016^{1}$, and eight of our sister journals have had this option since 2019. Starting this year, we join our sister journals Nature Methods and Nature Physics in offering this option to our authors. At Nature Computational Science, the decision to implement transparent peer review was a no-brainer as we see a number of immediate benefits to our community. First, this is a step towards making our editorial decision-making process open. Second, it provides greater visibility and recognition for peer reviewers and the incredible and essential work they do. Finally, the published reviews provide additional information and discussion on the paper, which adds more value to the published research.

At the same time that we want to ensure that the decision-making process for publishing a paper is open, we need to make sure that reviewers, authors and editors can communicate privately when necessary. Therefore, reviewers' comments exclusively directed to editors, correspondences between authors and editors, and all of the communication among the editors will remain confidential. Nevertheless, we will ensure that our decisions are clearly explained in the peer review document.

We believe this is an important step forward in the peer review process, and we hope that our authors, reviewers and readers will be as thrilled as we are in making research more transparent!

Published online: 25 March 2021

https://doi.org/10.1038/s43588-021-00054-7

References

1. Nat. Commun. 6, 10277 (2015). 